\title{
Ultrasonic velocity and attenuation in glass
}

\author{
U S GHOSH \\ Department of Solid State Physics, Indian Association for the Cultivation of Science, Calcutta \\ 700032 , India
}

\begin{abstract}
Ultrasonic velocity and attenuation in glasses exhibit anomalous properties and the current theory most successful in explaining these anomalous properties in the high temperature range has been presented. The model considers that the disorder inherent in amorphous network results in a double well potential corresponding to two equilibrium configurations and a particle is ascribed to move in the double-well potential. Acoustic behaviours in glasses have been interpreted in terms of a thermally activated relaxation process. Improvement of this basic model has also been discussed. Mention has also been made of some behaviours at high temperature that need further research for convincing explanation.
\end{abstract}

Keywords. Ultrasonic velocity and attenuation; double-well potential; thermally activated relaxation.

\section{Introduction}

For the last twentyfive years, studies on physical properties of glass have attracted the attention of solid state physicists mainly because glasses exhibit a number of properties strikingly different from their crystalline counterpart. In many cases, the differences become very prominent at extremely low temperature. These properties are: specific heat, thermal conductivity, thermal expansion, acoustic velocity, acoustic absorption, third order elastic constants, pressure coefficients of velocity to name a few (Krause and Kurkjian 1968; Hunklinger and Arnold 1976; Kul'bitskaya and Shutilov 1976; Anderson 1981; Pohl 1981; Hunklinger and Schickfus 1981).

In the present article, we shall confine our discussion to only within the behaviour of ultrasonic velocity and attenuation in glasses.

\section{Anomalous properties of acoustic velocity in glass}

For dielectric crystals, it is well known that

$$
\mathrm{d} C / \mathrm{d} T=0 \text { at } \quad T \approx 0,
$$

and $\mathrm{d} C / \mathrm{d} T$ is negative (magnitude very small) above $T=0$ where $T$ is the absolute temperature and $C$ the elastic constant. As a result similar variation is found in acoustic velocity as a function of temperature in dielectric crystals. The situation is quite different in glasses: (i) a strong variation with both positive and negative temperature coefficient of sound velocity is found between $0 \cdot 2 \mathrm{~K}$ and $5 \mathrm{~K}$ (Hunklinger and Arnold 1976; Hunklinger and Schickfus 1981). Within this temperature range the variation shows a maximum, (ii) beyond $5 \mathrm{~K}$ sound velocity falls gradually, attains a minimum between $50 \mathrm{~K}$ and room temperature depending on the nature of the glass and then rises with positive temperature coefficient (Hunklinger and Arnold 1976; Hunklinger and Schickfus 1981). At higher temperature (of course below $T_{\mathrm{g}}$, the glass transition temperature), the sound velocity rises almost linearly. The occurrence 
of minimum and subsequent rise of velocity with the temperature is found in all glasses containing high concentration of tetrahedral structures (Kul'bitskaya et al 1975; Hunklinger and Arnold 1976; Kul'bitskaya and Shutilov 1976; Hunklinger and Schickfus 1981), (iii) the value of sound velocity at high temperature before reaching $T_{\mathrm{g}}$ may considerably exceed the value at $0 \mathrm{~K}$ (Hunklinger and Arnold 1976; Hunklinger and Schickfus 1981), (iv) below $1 \mathrm{~K}$, dependence of velocity on temperature becomes logarithmic (Hunklinger and Arnold 1976; Hunklinger and Schickfus 1981) and (v) some glasses such as $\mathrm{B}_{2} \mathrm{O}_{3}, \mathrm{AS}_{2} \mathrm{~S}_{3}$ in which the random glass network is formed by planar units instead of three dimensional tetrahedral structures do not follow the variation indicated above in (ii) and (iii). Sound velocity falls monotonically with temperature in these cases (Hunklinger and Arnold 1976; Kul'bitskaya and Shutilov 1976; Hunklinger and Schickfus 1981).

\section{Anomalous properties of acoustic absorption}

Information regarding acoustic absorption is obtained from the measurement of attenuation of ultrasonic wave as it passes through the medium. It is known that for dielectric crystal ultrasonic attenuation at temperatures above $30 \mathrm{~K}$ for which $\omega \tau_{\mathrm{Th}} \ll 1$ is independent of temperature where $\omega$ is the frequency of the ultrasonic wave and $\tau_{T h}$ is the life time of the thermal phonons (Hunklinger and Arnold 1976). At low temperature for which $\omega \tau_{\mathrm{Th}} \gg 1$, the attenuation coefficient in dielectric crystal drops even faster than $T^{4}$ and becomes practically negligible below $10 \mathrm{~K}$ (Hunklinger and Arnold 1976; Hunklinger and Schickfus 1981). A strikingly different behaviour is found in glasses (Hunklinger and Arnold 1976; Hunklinger and Schickfus 1981). Not only that the magnitude of attenuation is much larger in glass than in crystal, the variation of ultrasonic attenuation with temperature shows a large and broad peak around $50 \mathrm{~K}$ or above depending on the nature of the glass. The peak value of attenuation is proportional to the frequency and the peak shifts slowly towards higher temperature with increasing frequency. A quadratic frequency dependence of absorption is found on the high temperature side of the peak. At low temperature around $5 \mathrm{~K}$ a second smaller peak or shoulder appears which shifts rapidly to higher temperature with increasing frequency. At still lower temperatures there is a steep decrease of absorption, the attenuation drops rapidly as $T^{\mathbf{3}}$ becomes frequency independent.

\section{Theoretical treatment}

The most successful model to explain the above mentioned low temperature anomalies in glass has been put forward by Anderson et al (1972) and Phillips (1972). The situation has been reviewed in a beautiful article by Jackle et al (1976). The model assumes the existence of an ensemble of two-level system (TLS) in glass with a flat distribution of level splitting. This also accounts for the presence of low energy excitations with approximately constant density of states in addition to phonons as indicated by the specific heat studies in glass, which show excess specific heat over that predicted by Debye theory. These TLS are considered as belonging to localized structural defects which at very low temperature perform quantum mechanical tunnelling motion through a barrier between two possible equilibrium configurations. 
Anderson and Bömmel (1955) indicated that in an amorphous network because of inherent disorder certain atoms or groups of atoms can occupy two different configurational states i.e. particles of unspecified nature constituting localized structural defects are able to move in a double well potential having two minima corresponding to two equilibrium configurations, and the two possible configurations of the defect in either of the two wells of the double well potential represent the two states of the defect. At low temperature only the two lowest energy levels with an energy separation determined by quantum mechanical tunnelling through the barrier are important. At high temperatures the two defect configurations should actually be treated as thermodynamical states involving a thermodynamical average over some vibrational degrees of freedom. At high temperature the double well potential should correctly be interpreted as free energy curve rather than potential energy curve.

At extremely low temperature when ultrasonic phonon interacts with TLS, transition between the two states are possible by tunnelling in which a resonant phonon is absorbed or emitted, this ultimately causes a net ultrasonic absorption which graduaily decreases with increase of the acoustic intensity and finally saturates due to increase of the population of upper level. At a slightly higher temperature, but still a very low temperature, another process of sound attenuation involving relaxation via tunnelling becomes important: the ultrasonic wave disturbs the thermal equilibrium of TLS, the disturbed TLS relaxes via the emission and absorption of resonant phonon.

At high temperature, a different process which can be described as a structural relaxation via thermally activated transition overriding the barrier height of the double-well potential becomes important. Thus, in glass two types of defects are distinguished: (1) tunnelling defect which is a structural defect but for which resonant absorption or emission of phonon occurs at very low temperature in a tunnelling process which may involve relaxation also and (2) thermally activated defects which are also structural defects but which when disturbed from its original state of thermal equilibrium by ultrasonic induced strain relaxes via thermally activated transition requiring higher temperatures. It is to be noted that both the types of defects are basically two state defects.

In the present article we are concerned with ultrasonic propagation behaviour in the high temperature range and hence we deal.with the thermally activated defects only. The thermally activated relaxation process has profound effect on ultrasonic velocity and attenuation in the higher temperature region and the observed behaviour in this temperature region are explained with the help of this relaxation process.

\section{A simple model for the microscopic origin of double well potential}

We now discuss a simple but interesting model given by Bridge and Patel (1986) regarding the microscopic origin of the two well potentials based on central force theory. Consider a three dimensional network of $\mathrm{A}-\mathrm{O}-\mathrm{A}$ bonds $(\mathrm{A}=$ cation, $\mathrm{O}=$ anion, for example an oxygen atom) in which $\mathrm{A}-\mathrm{O}-\mathrm{A}$ angles are not necessarily $180^{\circ}$. For a perfect crystalline arrangement all $\mathrm{A}-\mathrm{A}$ and $\mathrm{A}-\mathrm{O}$ separations (bond lengths) are the same and the central force theory predicts that all atoms of a particular kind move in identical symmetric interatomic potential wells each of which has a single central minimum for each atom corresponding to its equilibrium position and for sufficiently small vibrations, the motion of each atom is harmonic while at large 
amplitudes anharmonic effect appears causing flattening of the bottom of the well. This is the picture in crystalline arrangement. For the amorphous case there is always a distribution of $\mathrm{A}-\mathrm{A}$ separations (denoted by $\mathrm{R}$ ) with values both greater than or less than the equilibrium crystalline value. There may also be a distribution of $\mathrm{A}-\mathrm{O}$ bond lengths about the crystalline value. Bridge and Patel (1986) then developed their model for the microscopic origin of two well potentials in a glass network in the case of both longitudinal and transverse vibrations of the anions according to the simple classical central force theory, cations being considered to be too heavy to move. To illustrate the model we consider the simple case of longitudinal vibration of the anion ( $\mathrm{O}$ atom) in the linear system $\mathrm{A}-\mathrm{O}-\mathrm{A}$.

For a diatomic molecule of the type (A-O), the mutual potential energy $U$ at any instant is of the form

$$
U=-\frac{a}{r}+\frac{b}{r^{m}}
$$

where $6<m<12, r=\mathrm{A}-\mathrm{O}$ separation at any instant of motion, $a$ and $b$ are constants for a given molecular type which are obtained from the following relations, (i)

$$
\left(\frac{\mathrm{d} U}{\mathrm{~d} r}\right)_{r=r_{0}}=0
$$

which gives

and (ii)

$$
b=\left(a r_{0}^{m-1}\right) / m
$$

$$
U_{0}=\frac{a}{r_{0}}\left(1-\frac{1}{m}\right)
$$

where $U_{0}$ is the bond energy at the equilibrium interatomic separation $r_{0}$ (the crystalline value).

Next we consider the linear arrangement of three atoms (A-O-A) consisting of an anion in the middle of the two cations separated by a distance $R$. At any instant of motion of the anion, one A-O separation is denoted by $r$ and the other is $(R-r)$. We assume that the potential energy of the system at any instant is given by the superposition of two potentials of the form given by (2) i.e.

$$
U=\left(-\frac{a}{r}+\frac{b}{r^{m}}\right)+\left(-\frac{a}{R-r}+\frac{b}{(R-r)^{m}}\right) .
$$

If we define an elongation factor $e$ by $e=\left(R / 2 r_{0}\right)$ i.e. cation-cation separation divided by equilibrium separation $2 r_{0}$ (crystalline value), then

$$
U=-a\left[\frac{1}{r}+\frac{1}{2 e r_{0}-r}\right]+b\left[\frac{1}{r^{m}}+\frac{1}{\left(2 e r_{0}-r\right)^{m}}\right]
$$

The quantity $U / 2$ may be regarded as the mutual potential energy of half the anion atom plus one of the cation atoms and is taken as the potential in which the anion atom moves, each cation being considered infinitely heavy. It should be noted that direct 
interaction between the cation atoms has been ignored as it is a function of $R$ only and cannot affect the variation of $U$ with $r$ and is therefore of no interest to us.

Bridge and Patel (1986) considered the case of $\mathrm{P}-\mathrm{O}-\mathrm{P}$ system in $\mathrm{P}_{2} \mathrm{O}_{5}$ glass and calculated the variation of $U / 2$ with displacement of the $\mathrm{O}$-atom for various fixed values of $e$ using the $a$ and $b$ values evaluated from (4) and (5) and the known values of bond energy $U_{0}$ and bond length $r_{0}$ in $\mathrm{P}_{2} \mathrm{O}_{5}$ crystal and taking $m=9$. This is shown in figure 1 . It was observed that a single minimum in the potential energy occurs for $e<1$ and also for $e$ slightly greater than unity. However a two-well potential starts to develop for $e \geqslant 1 \cdot 2$ i.e. elongation of $\mathrm{P}-\mathrm{P}$ separation $(R)$ with respect to the crystalline value $2 r_{0}$ exceeds $20 \%$. For an elongation of $38 \%(e=1.38)$ a potential barrier of about $0.1 \mathrm{eV}$, the correct order of magnitude to explain the acoustic loss in $\mathrm{P}_{2} \mathrm{O}_{5}$ glass, occurs.

Similar calculation was carried out by Bridge and Patel (1986) for transverse displacement of the anion atom in the linear system $\mathbf{A}-\mathrm{O}-\mathrm{A}$ and it was found that for $e>1$, a single minimum in the potential occurs, but for $e<1$ two wells appear with a barrier of the right order of magnitude required to explain the acoustic loss for a small contraction (negative elongation) of $\sim 6 \%(e=0.94)$ in $\mathrm{P}_{2} \mathrm{O}_{5}$ glass. For the case of non- $180^{\circ} \mathrm{A}-\mathrm{O}-\mathrm{A}$ system in phosphate glass, Bridge and Patel (1986) further showed that the central force theory leads to double-well potential when $e$
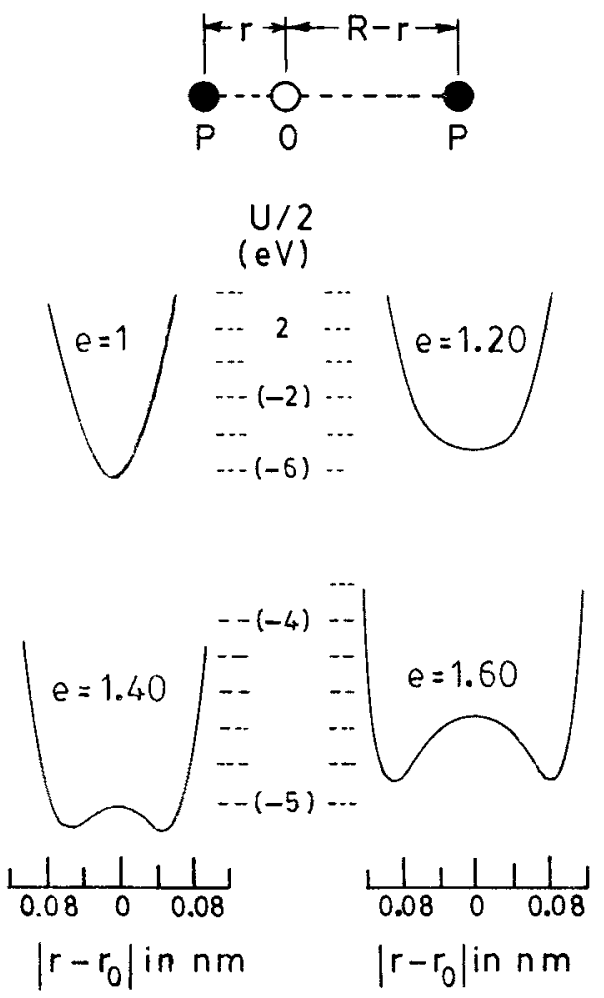

Figure 1. Potential wells for motion of oxygen atoms parallel to $\mathrm{P}-\mathrm{O}-\mathrm{P}$, in phosphorus pentoxide glass. 
values for the $\mathbf{P}-\mathbf{P}$ separation is greater than certain critical value for longitudinal motion and less than a different critical value for transverse motion.

\section{Relaxation effects on ultrasonic velocity and attenuation}

The relaxation effects of the defects in glass have their origin in the coupling of these defects with the elastic strain field. An ultrasonic wave through a glass causes an elastic strain which in turn causes a relative shift in energy (or free energy) between the two states of a defect. As a result the equilibrium of the defect is disturbed or in other words thermal inequilibrium takes place. A relaxation process sets in to restore the equilibrium. Now, relaxation is essentially a readjustment of occupation numbers of the two states of the defect (occupation number of a state is the probability with which the state is occupied). Therefore relaxation is described in terms of the time dependent occupation numbers $P_{1}$ and $P_{2}$ of the two states designated as states 1 and 2 , state 1 being considered to be higher in energy and because of the condition that $P_{1}+P_{2}=1$ always, a complete description of the two states can be given in terms of occupation number $P_{1}$ of state 1 alone. Denoting the equilibrium value of the occupation number by a superscript $(0)$, i.e. $P_{1}^{(0)}$ and $P_{2}^{(0)}$ for the two states and free energy of the two states before the application of the strain field by $F_{1}$ and $F_{2}$, we have

where

$$
\frac{P_{1}^{(0)}}{P_{2}^{(0)}}=\frac{e^{-\beta F_{1}}}{e^{-\beta F_{2}}}=e^{-\beta\left(F_{1}-F_{2}\right)}=e^{-\beta(\Delta F)},
$$

and

$$
\beta=\frac{1}{k T}, \quad k=\text { Boltzmann constant, } \Delta F=F_{1}-F_{2},
$$

$$
P_{1}^{(0)}+P_{2}^{(0)}=1
$$

From (8) and (9)

$$
P_{1}^{(0)}=\left[e^{\beta \Delta F}+1\right]^{-1}=f(\Delta F) \text { say. }
$$

The time dependence of the occupation number of the two states at any instant $t, P_{1}(t)$ and $P_{2}(t)$ are determined by the rate equation

$$
\frac{\mathrm{d}}{\mathrm{d} t} P_{1}(t)=-\mathrm{P}_{1}(t) W_{12}(t)+P_{2}(t) W_{21}(t)
$$

where $W_{12}$ and $W_{21}$ are the probabilities for transition per unit time from state 1 to state 2 and vice versa. W's may be time dependent because of the action of the time dependent perturbation by the acoustic field. An elastic strain $e(t)$ caused by the sound wave affects the two states of a defect and changes their free energies and also $\Delta F$ and hence their occupation numbers. We characterize this change of free energy difference for a strain of unit strength by a deformation potential $D$, defined by

i.e.

$$
\Delta F(e)=\Delta F+D \cdot e
$$

$$
D=\frac{\partial}{\partial e}[\Delta F(e)]
$$


In (12) $\Delta F$ refers to the equilibrium value. At every moment the relaxation processes aim at instantaneous equilibrium for which occupation number is not $P_{1}^{(0)}$ but $P_{1}^{(l)}$ defined by the occupation number that would be obtained at the equilibrium temperature for a free energy difference $\Delta F(e)$ (and not $\Delta F$ ) and analogous to (10) we will have

$$
\begin{aligned}
P_{1}^{(l)} & =f[\Delta F(e)]=f(\Delta F+D \cdot e) \\
& =f(\Delta F)+\frac{\partial f(\Delta F)}{\partial(\Delta F)} \cdot D \cdot e
\end{aligned}
$$

which gives the relation between the instantaneous and absolute equilibrium values of occupation number as

$$
P_{1}^{(l)}=P_{1}^{(0)}+\frac{\partial f(\Delta F)}{\partial(\Delta F)} \cdot D \cdot e .
$$

Our aim is to find the complete deviation of $\delta P_{1}$ of occupation number $P_{1}(t)$ at any instant $t$ from the absolute equilibrium value of $P_{1}^{(0)}$ i.e.

$$
\delta P_{1}=P_{1}(t)-P_{1}^{(0)} \text {. }
$$

Note that $P_{1}(t)$ refers to neither the instantaneous nor the absolute equilibrium value. Noting that the relaxation time is given by

$$
\tau^{-1}=W_{12}^{(0)}+W_{21}^{(0)},
$$

it can be shown [10] that

$$
\frac{\mathrm{d}}{\mathrm{d} t}\left[P_{1}(t)-P_{1}^{(0)}\right]+\frac{P_{1}(t)-P_{1}^{(0)}}{\tau}=\frac{1 \mathrm{~d} f(\Delta F)}{\tau \mathrm{d}(\Delta F)} D \cdot e,
$$

considering the time dependence of $e$ as $e \sim e^{-i \omega t}$ where $\omega$ is the frequency of the sound wave, the solution of (16) gives

$$
\delta P_{1}=P_{1}(t)-P_{1}^{(0)}=\frac{\mathrm{d} f(\Delta F)}{\mathrm{d}(\Delta F)} D \cdot e(1-i \omega \tau)^{-1} .
$$

This change of $\delta P_{1}$ in the occupation number causes a feed back on the elastic stress $\sigma$ and our next aim is to find this contribution of $\delta \sigma$ due to the change in $\delta P_{1}$ i.e. $\partial \sigma / \partial P_{1}$.

Now

$$
\sigma=\frac{1}{\Omega}\left(\frac{\partial \mathscr{F}}{\partial e}\right)_{T, P_{1}},
$$

where $\Omega$ is the volume of the undeformed solid and we have postulated a free energy function $\mathscr{F}$ of the whole system containing $N$ defects with $T, e, p$ as independent variables. The free energy function $\mathscr{F}$ of the whole system should not be confused with free energy $F_{1}$ or $F_{2}$ of the defect states

$$
\mathscr{F}\left(T, e, P_{1}\right)=\mathscr{F}^{\prime}\left(T, e, P_{1}\right)-T S\left(P_{1}\right),
$$


where $\mathscr{F}^{\prime}$ is the free energy function excluding entropy contribution $S\left(p_{1}\right)$ associated with the probability distribution of the defects between their two states. To find $\partial \sigma / \partial P_{1}$ we first find $\partial \mathscr{F}^{\prime} / \partial P_{1}$. We have

$$
\begin{aligned}
\mathscr{F}^{\prime} & =N P_{1} F_{1}+N P_{2} F_{2}=N P_{1}\left(F_{1}-F_{2}\right)+N F_{2}, \\
\frac{\partial \mathscr{F}^{\prime}}{\partial P_{1}} & =N\left(F_{1}-F_{2}\right)=N \Delta F, \\
\sigma & =\frac{1}{\Omega}\left(\frac{\partial \mathscr{F}}{\partial e}\right)_{T, P_{1}}=\frac{1}{\Omega}\left(\frac{\partial \mathscr{F}^{\prime}}{\partial e}\right)_{T, P_{1}} \\
\frac{\partial \sigma}{\partial P_{1}} & =\frac{1}{\Omega} \frac{\partial}{\partial P_{1}}\left(\frac{\partial \mathscr{F}^{\prime}}{\partial e}\right)=\frac{1}{\Omega} \frac{\partial}{\partial e}\left(\frac{\partial \mathscr{F}^{\prime}}{\partial P_{1}}\right)=\frac{1}{\Omega} \frac{\partial}{\partial e}(N \Delta F), \\
& =\frac{N}{\Omega} \frac{\partial}{\partial e}(\Delta F)=n D,
\end{aligned}
$$

where $n$ is the number of defects per unit volume.

Thus the defect contribution $\delta \sigma$ to the stress due to the change of the occupation number is given by (using (17) and (20))

$$
\delta \sigma=n D \delta P_{1}=n D^{2} \frac{\mathrm{d} f(\Delta F)}{\mathrm{d}(\Delta F)} e(1-i \omega \tau)^{-1}
$$

The dynamical contribution $\delta C(\omega)$ of the two state defects to the complex, frequency dependent elastic constant $C$ is

$$
\begin{aligned}
\delta C(\omega) & =\frac{\delta \sigma}{e}=n D^{2} \frac{\mathrm{d} f(\Delta F)}{\mathrm{d}(\Delta F)} \frac{1+i \omega \tau}{1+\omega^{2} \tau^{2}} \\
\operatorname{Re} \delta C(\omega) & =n D^{2} \frac{\mathrm{d} f(\Delta F)}{\mathrm{d}(\Delta F)} \frac{1}{1+\omega^{2} \tau^{2}} \\
\operatorname{Im} \delta C(\omega) & =n D^{2} \frac{\mathrm{d} f(\Delta F)}{\mathrm{d}(\Delta F)} \frac{\omega \tau}{1+\omega^{2} \tau^{2}}
\end{aligned}
$$

Where the complex elastic constant $C$ is written as

$$
C=C_{\infty}+\delta C(\omega)
$$

$\left(C_{\infty}\right.$ is the elastic constant without interaction of the defects with sound wave i.e. elastic constant at infinite relaxation time). From the relation $C_{\infty}=\rho v_{\infty}^{2}$,

$$
C=C_{\infty}+\delta C(\omega)=\rho\left[v_{\infty}+\delta v(\omega)\right]^{2}
$$

where $\rho$ is the density, it is easy to show that

$$
\operatorname{Re} \delta C(\omega)=2 \rho v_{\infty} \operatorname{Re} \delta v(\omega)
$$

where $v_{\infty}=$ velocity of sound wave without interaction i.e. at infinite relaxation time, 
$\operatorname{Re} \delta v(\omega)=$ Real part of the change of velocity from $v_{\infty}$ due to relaxation effect.

Experimentally it is the real part of velocity which is measured as phase velocity. Hence from (23) and (25), the relaxation effect on sound velocity (omitting the argument $\omega$ in $\delta v(\omega))$

$$
\delta v=\frac{\operatorname{Re} \delta C(\omega)}{2 \rho v_{\infty}}=\frac{1}{2 \rho v} n D^{2} \frac{\mathrm{d} f(\Delta F)}{\mathrm{d}(\Delta F)} \cdot \frac{1}{1+\omega^{2} \tau^{2}} \ldots
$$

(omitting the suffix $\infty$ in $v_{\infty}$ in the final expression).

Again, it can be shown that $\operatorname{Im} C$ which arises entirely from $\operatorname{Im} \delta C(\omega)$ in our case is related to the dissipation factor $Q^{-1}$ as follows:

$$
\begin{aligned}
Q^{-1}=\frac{2 \alpha v}{\omega} & =-\frac{1}{\rho v^{2}} \operatorname{Im} C=-\frac{1}{\rho v^{2}} \operatorname{Im} \delta C(\omega), \\
& =-\frac{1}{\rho v^{2}} n D^{2} \frac{\mathrm{d} f(\Delta F)}{\mathrm{d}(\Delta F)} \cdot \frac{\omega \tau}{1+\omega^{2} \tau^{2}}
\end{aligned}
$$

$(\alpha=$ attenuation coefficient $)$.

It should be noted that in (26) and (27) $v$ refers to the velocity without interaction of the sound wave with the defects and it is obtained from the experimental result of velocity at extremely low temperature (liquid helium) and $\delta v$ is the change of sound velocity from this value due to the interaction (i.e. relaxation effect). The variation $\delta v$ of the sound velocity and the dissipation factor $Q^{-1}$ arising out of the relaxation effect as given by (26) and (27) refer to one kind of defect only. Because of the amorphous phase there will be several kinds of defects having different values of $\tau$ which depends on activation energy $V$ (barrier height between the two wells of the double-well potential), and we will have a distribution function $P(V)$ of the activation energy $V$. Further, we have using (10)

$$
\frac{\mathrm{d} f(\Delta F)}{\mathrm{d}(\Delta F)}=-\beta \frac{e^{\beta \Delta F}}{\left(e^{\beta \Delta F}+1\right)^{2}}, \quad \beta=\frac{1}{k T} .
$$

In the temperature range of our interest $k T \gg \Delta F$ and

$$
\frac{\mathrm{d} f(\Delta F)}{\mathrm{d}(\Delta F)} \approx-\beta / 4=-\frac{1}{4 k T}
$$

The relaxation effects on sound velocity and absorption then become

$$
\begin{aligned}
\frac{\partial v}{v} & =-\frac{D^{2}}{8 \rho v^{2} k T} \int_{0}^{\infty} \frac{P(V) \mathrm{d} V}{1+\omega^{2} \tau^{2}}, \\
Q^{-1} & =\frac{D^{2}}{4 \rho v^{2} k T} \int_{0}^{\infty} \frac{P(V) \omega \tau}{1+\omega^{2} \tau^{2}} \mathrm{~d} V .
\end{aligned}
$$

Here $v$ refers to the sound velocity without interaction (i.e. at extremely low temperature say liquid $\mathrm{He}$ ). 


\section{Expression for the relaxation time}

For the evaluation of (28) and (29) we need an expression for $\tau$. From reaction rate theory, rate of an activated process is proportional to $e^{-\beta\left(F_{a}-F_{i}\right)}$ where $F_{a}$ is the reaction threshold and $F_{i}$ the free energy of the initial state. The transition probabilities $W_{12}$ and $W_{21}$ are then

$$
\begin{aligned}
& W_{12}=\omega_{a 1} e^{-\beta\left(F_{a}-F_{1}\right)}, \\
& W_{21}=\omega_{a 2} e^{-\beta\left(F_{a}-F_{2}\right)} .
\end{aligned}
$$

At equilibrium

and

$$
P_{1}^{(0)} W_{12}^{(0)}=P_{2}^{(0)} W_{21}^{(0)}
$$

$$
\frac{P_{1}^{(0)}}{P_{2}^{(0)}}=\frac{e^{-\beta F_{1}}}{e^{-\beta F_{2}}}
$$

Using these equations at equilibrium, we have

and

$$
\omega_{a 1}=\omega_{a 2}=\omega_{a}^{(0)} \mathrm{say}
$$

$$
\frac{1}{\tau}=W_{12}^{(0)}+W_{21}^{(0)}=W_{a}^{(0)} e^{-\beta\left(F_{a}-F_{i}\right)}\left[1+e^{-\beta\left(F_{1}-F_{2}\right)}\right]
$$

The energy difference $\left(F_{a}-F_{i}\right)$ corresponds to barrier height $V$, hence

$$
\tau=\tau_{0} e^{V / k T}\left(1+e^{-(\Delta F / k T)}\right)^{-1}, \quad \tau_{0}=\frac{1}{\omega_{a}^{(0)}} .
$$

At high temperature for which $\Delta F \ll k T$,

$$
\tau=\tau(0) e^{V / k T}, \quad \tau(0)=\frac{1}{2} \tau_{0}
$$

\section{Improvement of the theory}

Earlier theoretical works using (28), (29) and (31) or (30) considered (a) a symmetric double-well potential for which the two minima were equal in energy, (b) a gaussian distribution $P(V)$ for the barrier height only, (c) a low barrier cut-off is needed to produce a peak in $Q^{-1}$. In vitreous silica, a cut-off for $V$ below $125 \mathrm{~K}$, and a width $\sim 410 \mathrm{~K}$ of the gaussian distribution are needed for agreement with experiment and (d) frequency dependence of attenuation was not satisfactory.

The theory has been improved by Gilroy and Phillips (1981) so as to give a very satisfactory agreement with the experiment. The model is based on an asymmetric double well potential having distribution for both barrier height and asymmetry. The model has the advantage that no low barrier cut off is required contrary to the symmetric double well model. The same variations in local environment that produce variations in the barrier height in glass are also expected to produce inequivalent 
potential wells having unequal depths in the double well potential curve. Thus the improved theory considered (i) asymmetry parameter $\Delta$ of the wells given by $\Delta=$ the difference in energy of the two potential minima and a distribution of $\Delta$ denoted by $n(\Delta)$, (ii) no lower cut off for $V$, (iii) distribution of $V$ of the form $g(V)=\left(1 / V_{0}\right) e^{-V / V_{0}}$ where $V_{0}$ is the energy value of the order that corresponds to glass transition temperature $T_{\mathrm{g}}$. Gilroy and Phillips $(1981)$ choose $n(\Delta)$ as a constant $n_{0}$, valid for temperature below $T_{\mathrm{g}}$ and (iv) further in their analysis relaxation time $\tau$ was expressed as

$$
\tau=\tau_{0} e^{V / k T} \sec h(\Delta / 2 k T)
$$

which is a natural consequence of the introduction of asymmetry in (30). With the introduction of asymmetry, (30) may be written as

$$
\tau=\tau_{0} e^{(V-\Delta / 2) / k T}\left(1+e^{-(\Delta / k T)}\right)^{-1}
$$

where $V$ is now the barrier height from the lower well, $V-\Delta / 2$ is the average of the barrier heights measured from the two inequivalent wells and $\Delta F$ should be replaced by $\Delta$ when asymmetry parameter is taken into consideration. It is worthwhile to note that (33) can be shown to be identical in form with (32).

Improved theory of Gilroy and Phillips (1981) gives

$$
\begin{aligned}
\frac{\delta v}{v} & =-\frac{D^{2}}{8 \rho v^{2} k T} \int_{0}^{\infty} \int_{0}^{\infty} \frac{1}{1+\omega^{2} \tau^{2}} \sec h^{2}\left(\frac{\Delta}{2 k T}\right) n(\Delta) g(V) \mathrm{d} \Delta \mathrm{d} V, \\
Q^{-1} & =\frac{D^{2}}{4 \rho v^{2} k T} \int_{0}^{\infty} \int_{0}^{\infty} \frac{\omega \tau}{1+\omega^{2} \tau^{2}} \sec h^{2}\left(\frac{\Delta}{2 k T}\right) n(\Delta) g(V) \mathrm{d} \Delta \mathrm{d} V .
\end{aligned}
$$

Gilroy and Phillips (1981) gave the approximate solution of (34) and (35) in closed form as follows

$$
\begin{aligned}
\frac{\delta v}{v} & =-\frac{D^{2}}{4 \rho v^{2}} n_{0}\left[1-\left(\omega \tau_{0}\right)^{k T / V_{0}}\right], \\
Q^{-1} & =\frac{D^{2}}{4 \rho v^{2}} n_{0} \pi \frac{k T}{V_{0}}\left(\omega \tau_{0}\right)^{k T / r_{0}} .
\end{aligned}
$$

$Q^{-1}$ as a function of temperature shows a maximum when $\mathrm{d} Q^{-1} / \mathrm{d} T=0$ which leads to

i.e.

$$
\omega \tau_{0}=e^{-\left(V_{0} / k T_{m}\right)}
$$

$$
\ln \omega+\ln \tau_{0}=-\frac{V_{0}}{k T_{m}} .
$$

Where $T_{m}$ is the temperature at which the $Q^{-1}$ peak occurs and

$$
Q_{\max }^{-1}=\frac{\pi n_{0} D^{2} k T_{m}}{4 \rho v^{2} V_{0} e}
$$

The relaxation effects on both the sound velocity $(\delta v / v)$ and dissipation $\left(Q^{-1}\right)$ contain the same parameters such as $\left(n_{0} D^{2}\right) / \rho v^{2}, \tau_{0}$ and $V_{0}$. While $Q^{-1}$ is obtained from the 
attenuation coefficient which is directly measured in the ultrasonic attenuation experiment, the relative variation of velocity $\delta v / v$ at any temperature $T$ is usually obtained from the velocity measurements $v_{T}$ at temperature $T$ and $v_{\mathrm{He}}$ at the temperature of liquid helium where relaxation effect is practically nil. i.e.

$$
\frac{\delta v}{v}=\frac{v_{T}-v_{\mathrm{He}}}{v_{\mathrm{He}}}
$$

Gilroy and Phillips (1981) were able to fit their theory with experiments on vitreous silica by adjusting suitable values of these parameters consistent with other experiments. However, the parameters $\tau_{0}$ and $V_{0}$ can be obtained from a plot of $\ln \omega$ vs $1 / T_{m}$ if attenuation experiments are performed at different frequencies. From (38) the plot should be a straight line which should yield $\ln \tau_{0}$ and $V_{0} / k$ from its intercept and slope. Bridge and Patel (1986) in their studies on ultrasonic relaxation in molybdenumphosphate glasses of different composition also used the expression of $Q^{-1}$ analogous to (35), but instead of going for an analytic solution they adopted a method of numerical computation of the relevant integrals in order to fit the experimental $Q^{-1}$ curve. Recently Mukherjee et al (1993) carried out ultrasonic investigation in the binary system of $\mathrm{V}_{2} \mathrm{O}_{5}-\mathrm{GeO}_{2}$ glasses of different compositions and evaluated the parameters necessary to interpret the attenuation $\left(Q^{-1}\right)$ experiment successfully following Gilroy and Phillips (1981) theory.

\section{Present status of the theory}

It is worthwhile to mention here that Gilroy and Phillips (1981) had to consider in their theory for the variation of velocity, additional term to account for the high temperature velocity variation which cannot be explained by the relaxation effects. It should be noted that (36) indicates a negative value of $\delta v$ i.e. sound velocity decreases due to relaxation effect from its value without relaxation (the negative value of $\delta v$ is apparent if we note that $\left.\omega \tau_{0} \ll 1\right)$ and the magnitude of this decrease in $\delta v$ increases as $T$ increases. Hence the linear rise of velocity with temperature at higher temperature cannot be explained by this theory, nor can it explain a value of velocity at high temperature greater than that without relaxation effect i.e. greater than the value at extremely low temperature (say He temperature). This is why Gilroy and Phillips (1981) had to consider additional term indicating a rise of velocity with temperature observed in quartz glass. While the theory of the relaxation effects on attenuation (i.e. $Q^{-1}$ ) as given by (37) adequately explains the attenuation behaviour of glass, the theory can explain the initial decrease of $(\delta v / v)$ with increasing temperature in the region below the temperature at which the minimum occurs but cannot account for the almost linear rise on the higher temperature side without including a suitable term as mentioned above. In the case of vitreous silica attempt has been made by Dienes (1958) to relate this increase of $v$ with $T$ at higher temperature to the anomalously small thermal expansion coefficient of glass compared to that of crystalline counterpart. The small thermal expansion coefficient of vitreous silica leads to positive temperature coefficient of elastic moduli and hence a positive temperature coefficient of velocity. But such explanation in terms of small thermal expansion fails in many other glasses where thermal expansion is not small but comparable to the value for their crystalline 
counterpart. Another explanation has been suggested by Kul'bitskaya et al (1975, 1976) in terms of the behaviour of microscopic elastic inhomogeneities that exist in a glass and are associated with the freezing of natural fluctuations of the order parameter of liquid glass at temperatures close to $T_{\mathrm{g}}$. When the rate of cooling is high, the viscosity of a glass forming substance may be so high that the order fluctuations are not dispersed but are frozen at temperature $T_{\mathrm{g}}$ and are thus retained in the solid state. Moreover, cooling below $T_{\mathrm{g}}$ may further increase the tensile stresses between the regions of higher and lower order i.e. it may enhance the local elastic inhomogeneities. Kul'bitskaya et al $(1975,1976)$ showed that the effective macroscopic elastic constant $C_{\text {eff }}$, which governs the velocity of propagation of ultrasonic wave in such an elastically inhomogeneous medium, is equal to an average value of $C_{0}$ from which is subtracted a positive quantity $\Delta_{f}^{2}$ proportional to the mean square amplitude of the spatial fluctuations of the elasticity i.e.

$$
C_{\text {eff }}=C_{0}-\Delta_{f}^{2}
$$

With the increase of temperature both $C_{0}$ and $\Delta_{f}^{2}$ decreases. If the correction $\Delta_{f}^{2}$ decreases more rapidly than $C_{0}$ as the temperature is increased, resulting in homogenization of glass structure, the macroscopic elastic constant $C_{\text {eff }}$ may increase producing positive temperature coefficient of velocity. The above mechanism of the formation of microscopic elastic inhomogeneity in glasses suggest that the temperature dependence of $\Delta_{f}^{2}$ should be more pronounced in high $T_{\mathrm{g}}$ glasses and this is why some doubt has arisen about the validity of this mechanism in the case of $\mathrm{BeF}_{2}$ where $T_{\mathrm{g}}$ is small, $592 \mathrm{~K}$ (i.e. slightly greater than that of $\mathrm{B}_{2} \mathrm{O}_{3}$ for which $T_{\mathrm{g}}=513 \mathrm{~K}$ and temperature coefficient of velocity is always negative), yet showing a positive temperature coefficient of velocity even more pronounced than in vitreous silica. The situation regarding positive temperature coefficient of velocity in the high temperature region observed in many glasses is not yet fully clear and is still of theoretical interest.

\section{References}

Anderson A C 1981 in Amorphous solids, low temperature properties (ed.) W A Phillips (Berlin: Springer Verlag) p. 27

Anderson O L and Bömmel H E $1955 \mathrm{~J}$. Ceram. Soc. 38125

Anderson P W, Halperin B I and Verma C 1972 Philos. Mag. 251

Bridge B and Patel N D 1986 J. Mater. Sci. 213783

Dienes G J 1958 J. Phys. Chem. Solids 7290

Gilroy K S and Phillips W A 1981 Philos. Mag. B43 735

Hunklinger S and Arnold W 1976 Phys. Acoust. 12155

Hunklinger S and Schickfus M V 1981 in Amorphous solids, low temperature properties (ed.) W A Phillips (Berlin: Springer Verlag) p. 91

Jackle J, Piche L, Arnold W and Hunklinger S 1976 J. Non-Cryst. Solids 20365

Krause J T and Kurkjian C R 1968 J. Am. Ceram. Soc. 51226

Kul'bitskaya M N and Shutilov V A 1976 Sov. Phys. Acoust. 22451

Kul'bitskaya M N, Nemilev S V and Shutilov V A 1975 Sov. Phys. Solid State 162319

Mukherjee S, Maiti A, Ghosh U S and Basu C 1993 Philos. Mag. B67 823

Phillips W A 1972 J. Low Temp. Phys. 7351

Pohl R O 1981 in Amorphous solids, low temperature properties (ed.) W A Phillips (Berlin: Springer Verlag) p. 27 